\title{
ASSOCIATION OF PHYSICAL ACTIVITY AND ACADEMIC PERFORMANCE IN SCHOOLCHILDREN OF NEPAL
}

\author{
Ram Manohar Basnet, Laxman Manohar Basnet \\ Health Concern, Ratopul, Kathmandu, Nepal
}

\begin{abstract}
Background. There is growing interest in the association between physical activity (PA) and academic performance (AP) in children, especially in the developing world. However, no in-depth study of such kind has yet been undertaken in Nepal. Therefore, this study was carried out to determine if there is correlation between PA and AP in secondary schoolchildren in Nepal.

Methods. A crosssectional study was carried in four schools of Nepal. Two schools (one private and one public) were randomly selected from two different districts. Physical activity and academic performance levels of students were obtained via questionnaires and Pearson's correlation was done to determine any association.

Results. Our study showed a significant correlation between physical activity and academic performance in the study population. The correlation was significant for male students, students from rural and urban areas and from public schools. However, there was no significant correlation in the female population and students of private schools. We also observed a significant difference in the physical activity performed by male versus female students (male $>$ female), along with a significant difference in their academic performance (male $>$ female). Further analysis of correlation on the basis of gender showed that physical activity of male students was significantly correlated with the academic performance irrespective of the location and type of school. However there was no such correlation in the female students.

Conclusion. Our study found that male students who were physically active showed positive correlation between physical activity and academic performance while no such correlation was found in the female students.
\end{abstract}

Keywords: physical activity, academic performance, schoolchildren, Nepal.

\section{INTRODUCTION}

$\mathrm{T}$ The World Health Organization (WHO) defines physical activity (PA) as any bodily movement produced by skeletal muscles that requires energy expenditure (World Health Organization, 2017b). Highlighting the importance of PA in the prevention of non-communicable diseases, WHO has developed a recommendation strategy entitled "Global Recommendations on Physical Activity for Health", which addresses three age groups: 5-17 years old, 18-64 years old and 65 years old and above (World Health Organization, 2017a).

PA is vital in improving general circulation, increasing blood flow to the brain, as well as raising norepinephrine and endorphin levels.
These all play a role in reducing stress, improving mood, and inducing a calming effect, therefore it could be argued that PA is helpful in improving achievement. Adolescents that are physically active are less likely to attempt suicide and are more likely to adopt risk-taking behaviours that may have an association with better academic outcomes (Taras, 2005). Regular participation in sport activities also results in improved behaviour in the classroom and aids concentration required during academic lessons (Singh, Uijtdewilligen, Twisk, van Mechelen, \& Chinapaw, 2012).

Prior to 2005, limited studies were done analysing PA and its association with academic performance (AP) (Taras, 2005). However, after 
2005, research in this domain gained significant momentum and currently extensive research has been done on this topic around the world. A recent study by Fox, Barr-Anderson, Neumark-Sztainer, and Wall (2010) found positive associations between PA involvement and AP among middle school and high school students, and further studies were recommended to understand the complex nature of this association. Singh et al. (2012) drew from their research and also found that participation in PA is positively related to AP in children, with similar results also found by various other studies (Booth et al., 2013; Correa-Burrows, Burrows, Ibaceta, Orellana, \& Ivanovic, 2014; Janak et al., 2014; Stea \& Torstveit, 2014). Some researchers have also studied the correlation between physical inactivity and academic achievements. A study by Shin and So (2012), analyzing the association between physical inactivity and academic record in Korean adolescents, concluded that Korean adolescents who spend more time engaged in physical inactivity are predisposed to a below average academic record.

However, not all studies have shown positive associations between PA and AP. Research done by LeBlanc et al. (2012) showed that increased PA did not result in significantly improved AP. Additionally, Esteban-Cornejo et al. (2014) found that PA might influence AP in both children and adolescents, but this association was in fact negative and very weak. Likewise, a study done in the United States found a positive association of PA and AP among females, while no such association was found among males (Carlson et al., 2008).

The purpose of our research was to determine if an association existed between PA and AP among secondary schoolchildren in Nepal. Nepal has four major topographical zones: the mountains, the hills, the valleys, and the terrain. This diversity in geography has given way to the creation of various cultures and social groups (Pokharel \& Poudel, 2013). Additionally, this diversity, coupled with a weak economic resource base, as well as inefficient and poor mobilization of available resources, has created extreme social and economic inequalities (Sharma, 1989-1992), which can greatly impact the quality of education a child receives (Devkota \& Bagale, 2015).

As of yet, no study has been undertaken in Nepal to understand if an association between PA and AP exists. Our research will therefore open academic discussions unique to this part of the world. We first aimed to understand the intensity of PA students partake in during school, after school and during the weekends. Secondly, by comparing academic outcomes with the frequency that students perform PA, we aimed to identify if there was any significant relationship between PA and AP.

\section{METHODS}

Participants. A total of 419 school students were selected for the study, all aged between 12 16 years and studying in the 8th and 9th grade. Thirteen students were excluded due to incomplete and/or incorrectly filled responses, and therefore 406 students participated in the study. The schools were selected based on public/private and urban/ rural areas. Four schools were selected, two each from two different districts: two schools (one government and one private) from the Kathmandu district (urban area) and two other schools (one government and one private) from the Dhading district (rural area). According to the United Nations Development Programme (UNDP), the human development index (HDI) of Kathmandu is $0.632(>0.550)$ and that of Dhading is $0.450-0.459$, while the overall HDI of Nepal is 0.458 (United Nations Development Programme and National Planning Commission, 2014). Our chosen study areas included districts that have slightly lower and higher HDIs than the average national HDI of Nepal. $40.1 \%$ of the students were studying in rural area (Dhading) and the remaining 59.8\% were studying in an urban area (Kathmandu).

Study Design. We conducted a cross-sectional study of Nepalese schoolchildren in 2014. For our study, we prepared a questionnaire (see supplementary information for full questionnaire) which consisted of two parts: the first part contained questions about the students' PA levels and the second part contained questions about AP. The first part of the questionnaire was adopted from Kowalski, Crooker, and Donen (2004). The second part of the questionnaire was crosschecked with the respective class teachers, after receiving the required permission from guardians and the respective schools. A formal consent was also obtained from the school administration of each participating school, prior to data collection, and only those students willing to volunteer in the study were included. Before handing out the questionnaires, students were first informed about the nature of the research.

Upon distributing the questionnaires, in order to avoid any confusion, all questions were orally explained to the students. The first part of the 
Table 1. Part one: questions on physical activity levels in the past seven days

\begin{tabular}{|c|c|}
\hline Questions & Answer options \\
\hline $\begin{array}{l}\text { In the last } 7 \text { days, during your physical education (PE) } \\
\text { classes, how often were you very active (playing hard, } \\
\text { running, jumping)? }\end{array}$ & $\begin{array}{l}\text { a) We don't have PE; b) Hardly ever; c) Sometimes; d) Quite } \\
\text { often; e) Always }\end{array}$ \\
\hline $\begin{array}{l}\text { In the last } 7 \text { days, what did you do most of the time at your } \\
\text { spare time at school? }\end{array}$ & $\begin{array}{l}\text { a) Sat down (talking, reading, doing schoolwork); b) Stood around } \\
\text { or walked around; c) Ran or played a little bit; d) Ran around and } \\
\text { played quite a bit; e) Ran and played hard most of the time }\end{array}$ \\
\hline $\begin{array}{l}\text { In the last } 7 \text { days, after school on how many times did you do } \\
\text { sports, dance, or play games in which you were very active? }\end{array}$ & $\begin{array}{l}\text { a) None; b) } 1 \text { time; c) } 2 \text { to } 3 \text { times; d) } 4 \text { to } 5 \text { times; e) } 6 \text { or more } \\
\text { times }\end{array}$ \\
\hline $\begin{array}{l}\text { In the last } 7 \text { days, on how many evenings did you do sports, } \\
\text { dance, or play games in which you were very active? }\end{array}$ & $\begin{array}{l}\text { a) None; b) } 1 \text { time; c) } 2 \text { to } 3 \text { times; d) } 4 \text { to } 5 \text { times; e) } 6 \text { or more } \\
\text { times }\end{array}$ \\
\hline $\begin{array}{l}\text { On the last weekend, how many times did you do sports, } \\
\text { dance, or play games in which you were very active? }\end{array}$ & $\begin{array}{l}\text { a) None; b) } 1 \text { time; c) } 2 \text { to } 3 \text { times; d) } 4 \text { to } 5 \text { times; e) } 6 \text { or more } \\
\text { times }\end{array}$ \\
\hline
\end{tabular}

questionnaire was modified from the documents in the study by Kowalski et al. (2004). Five questions were used for evaluating the physical activity of a subject in the last seven days. Each question was close-ended and scaled in the Likert scale from the lowest to the highest level of PA. Each student was asked about the physical activity he/she performed during the period of last seven days (Table 1).

After obtaining the student responses, the PA level of each student was quantified, to obtain each student's PA score. For this reason, we assigned specific points to each option of the questionnaires. The lowest level of physical activity (option a) was assigned 20 points, following with 40 (option b), 60 (option c), 80 (option d) to a maximum of 100 points (option e). The PA score of each student was calculated after taking the mean of the cumulative scores obtained from the responses to these five questions.

The academic performance of the students was self-reported within the questionnaires. In order to assist with recalling their scores, students were given the option to report their AP in ranges: less than 50, 50-60, 60-70, 70-80, 80-90 and $>90 \%$. Students were asked to report their AP of the year 2013, as the 2014 session was just starting during the research period. The overall academic score of the year 2013, along with scores in science, mathematics, and languages (English and Nepali) were also obtained for reference, although we did not consider the subject scores in science, mathematics and languages for this study. The AP reported from the students was then cross-checked with the help of their respective class teachers. Any incompletely and incorrectly filled questionnaires were excluded from the study. For data analysis purposes, AP less than $50 \%$ was scored as 1 ,
$50-60 \%$ was scored as $2,60-70 \%$ was scored as 3 , $70-80 \%$ was scored as $4,80-90 \%$ was scored as 5 and $>90 \%$ was scored as 6 .

Statistical Analysis. Pearson's correlation analysis was used to study the correlation between $\mathrm{PA}$ and AP in the school children, using a number of variables such as gender, public/private, urban/ rural. Two-tailed $t$-test was used to see if there were any significant differences in PA performed by male and female students and their AP. All data analyses were done using GraphPad Prism 5, La Jolla California USA. The alpha value for both the correlation and significance test was .05 .

\section{RESULTS}

Our study found a significant association between physical activity and academic performance in the total study population $(r=$ $.2158, p<.0001)$. The association were significant for male students $(r=.296, p<.0001)$, for students from rural areas $(r=.1877, p=.0164)$ as well as for students from urban areas $(r=.2276, p=.0003)$ and for students from public schools $(r=.3125$, $p<.0001)$. However, there was no significant correlation between PA and AP found in the female population $(r=.1199, p=.0793)$ and among students from private schools $(r=.1246, p=.1275)$. Also, linear regression analysis showed that the line of goodness of fit obtained was significantly non-linear in the overall population $\left(r^{2}=.0465, p<\right.$ $.0001)$, in the male students $\left(r^{2}=.0875, p<.0001\right)$, rural students $\left(r^{2}=.0352, p=.0161\right)$ and urban students $\left(r^{2}=.0518, p=.0003\right)$ and students from public schools $\left(r^{2}=.0976, p<.0001\right)$. The results of the correlation analysis are tabulated in Table 2 and graphically shown in Figure 1. 
Table 2. Correlation between physical activity (PA) and academic performance (AP) of overall population and different subgroups

Notes. $n$ - number of sample size; $r$-correlation coefficient; $r^{2}-$ goodness of fit.

\begin{tabular}{|l|c|c|c|c|}
\hline & $\boldsymbol{r}$-value & $\boldsymbol{p}$-value & $\boldsymbol{r}^{\mathbf{2}}$ & $\boldsymbol{p}$-value \\
\hline Overall $(n=406)$ & .2158 & $<.0001$ & .04658 & $<.0001$ \\
\hline Female $(n=215)$ & .1199 & .0793 & .01438 & .0793 \\
Male $(n=191)$ & .296 & $<.0001$ & .08759 & $<.00001$ \\
\hline Rural $(n=163)$ & .1877 & .0164 & .03524 & .0161 \\
Urban $(n=243)$ & .2276 & .0003 & .05181 & .0003 \\
\hline Public $(n=255)$ & .3125 & $<.0001$ & .09764 & $<.0001$ \\
Private $(n=151)$ & .1246 & .1275 & .01552 & .01275 \\
\hline
\end{tabular}

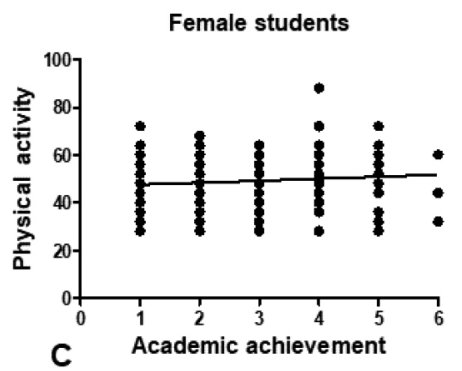

C Academic achievement

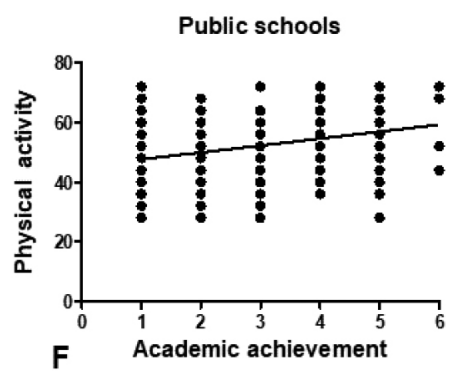

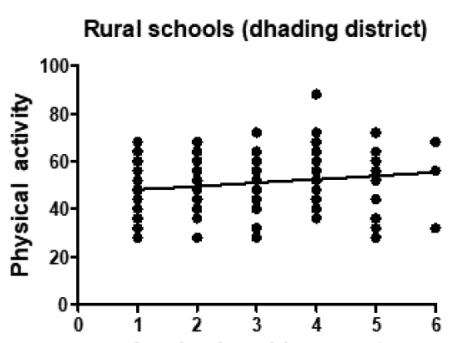

D Academic achievement

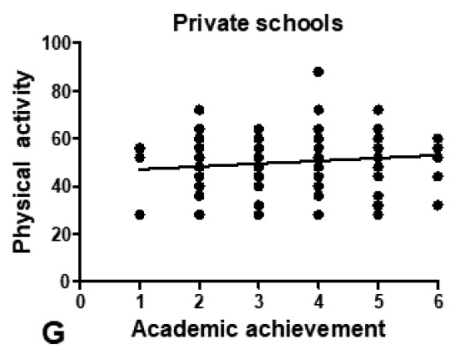

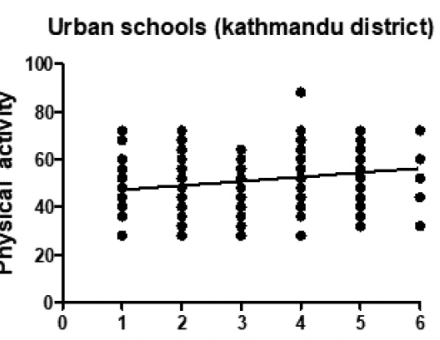

E Academic achievement

Figure 1. (A) Correlation between physical activity (PA) and academic performance (AP) in all school children; (B) male students; (C) female students; (D) rural school children; (E) urban school children; (F) public school; (G) private school

Note. $\mathrm{PA}$ - physical activity; $\mathrm{AP}$ - academic performance.

The average PA performed by males was 53.05 while that of females was 49.04, out of a maximum amount of 100 and a minimum of 20. Similarly, the average AP in males was 3.15 and in females was 2.84 , with a maximum point of 6 and a minimum of 1 (Figure 2). We performed a significance test to see whether there was any significant difference between PA of males and females, as well as a significant difference in their respective AP. Two tailed $t$-test showed that there was a significant difference in the PA levels of males and females ( $p=.0001)$ as well as a significant difference in their $\operatorname{AP}(p=.02)$

As there was a significant difference in the $\mathrm{PA}$ and AP of the male and female students, we did a further statistical analysis and performed the correlation analysis on the gender basis. The 


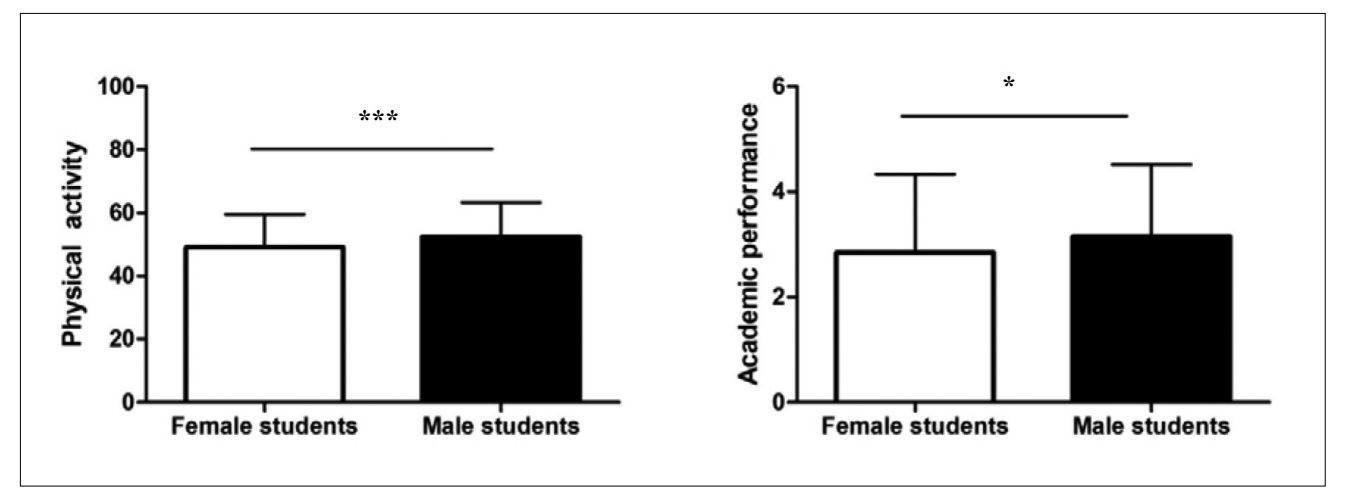

Figure 2. Left: physical activity performed by the female and male students. Right: academic achievement of female and male students. Asterisk indicates a significant difference between the male and female students $(* p \leq .05, * * *$ $p \leq .001)$

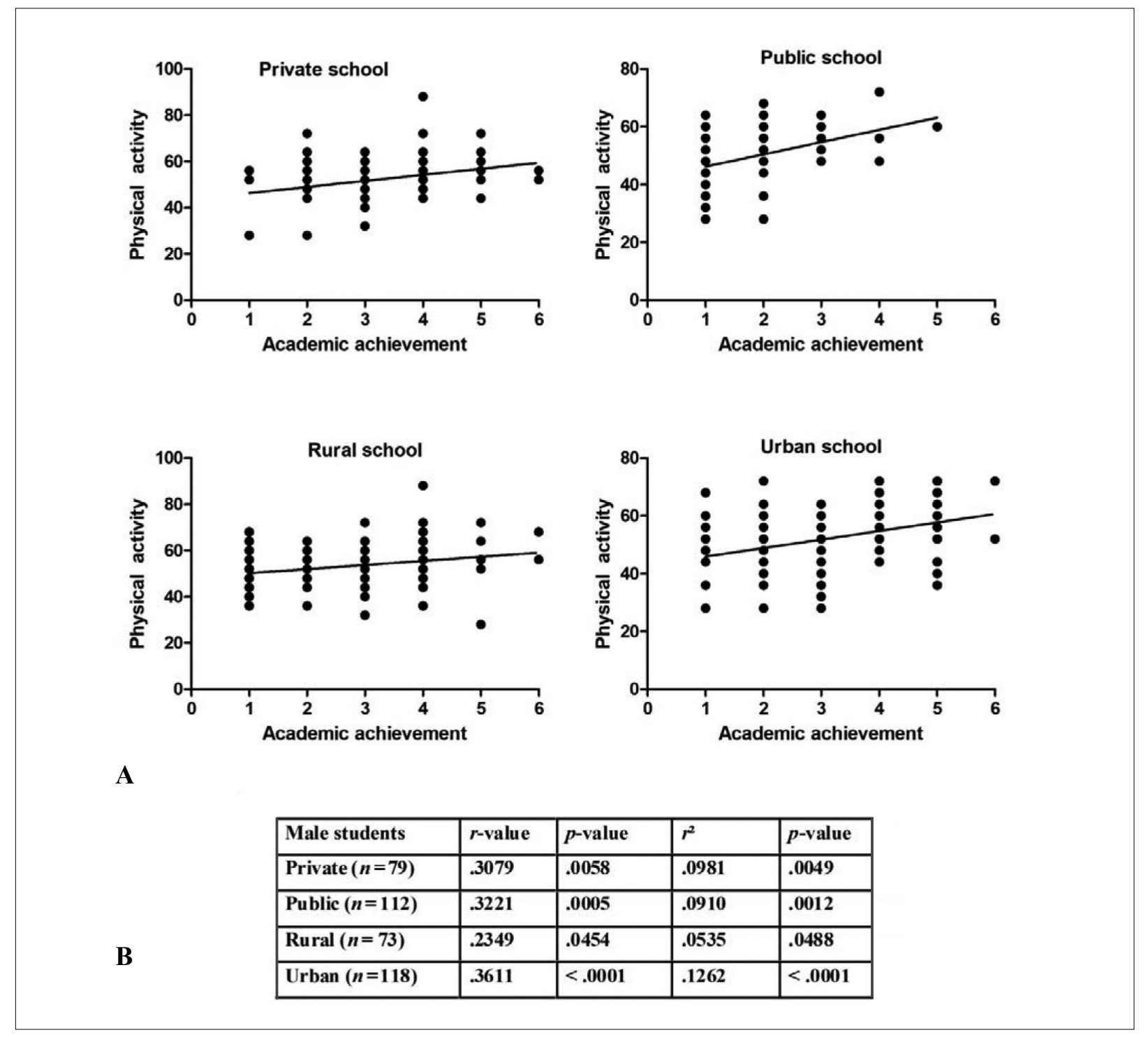

Figure 3. A-Correlation between physical activity (PA) and academic performance (AP) of male students in private, public, rural and urban Schools. B-corresponding table illustrating spearman correlation coefficient $(r)$ and goodness of fit $\left(r^{2}\right)$

Note. $n$ - number of students.

correlation of PA and AP was obtained for the private, public, rural and urban school for each group of male and female students. We found that PA and AP correlated positively in the male students irrespective of the type and location of school (Figure 3) whereas in case of female students there was significant correlation only in the female students of public schools (Figure 4). 


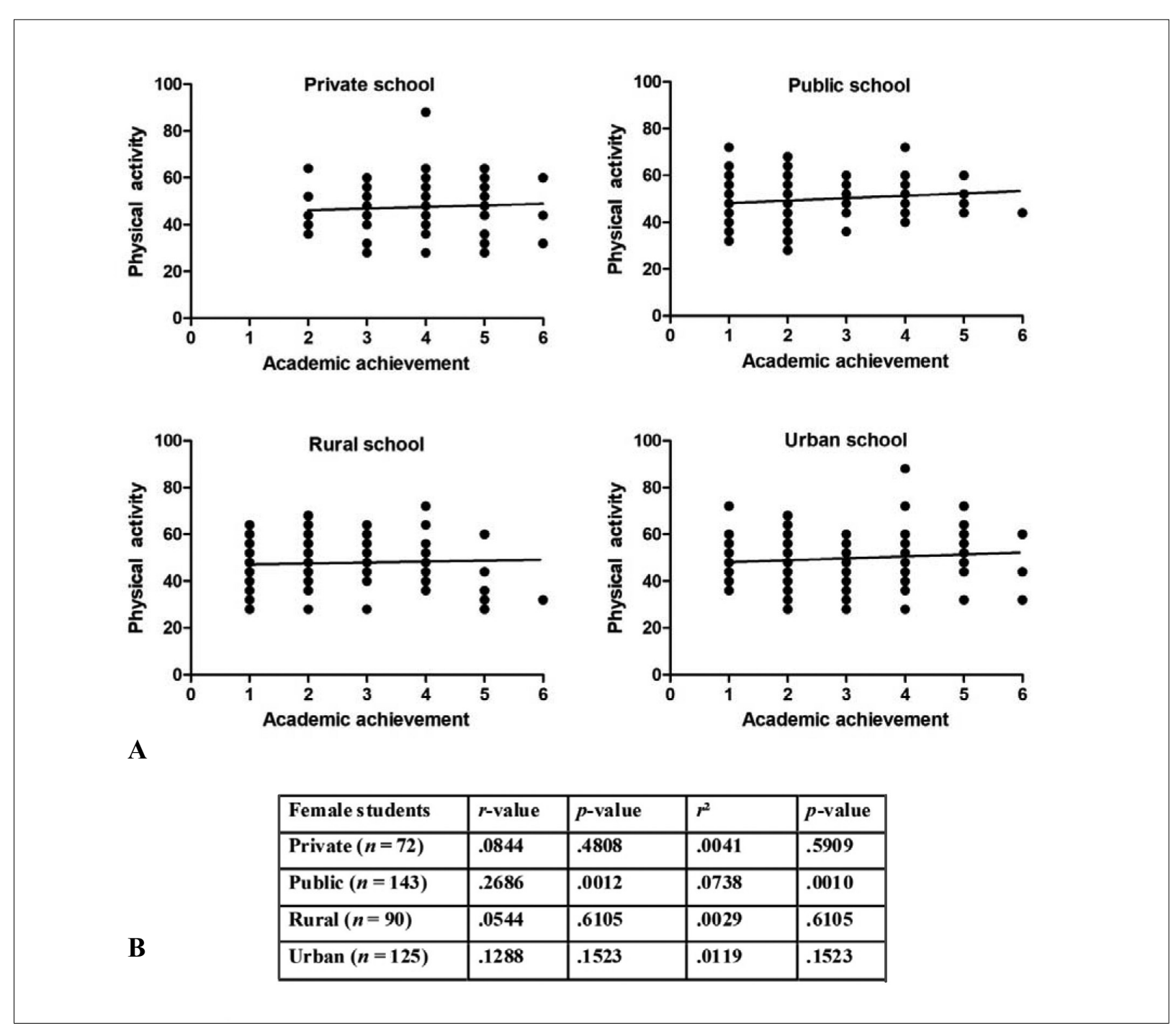

Figure 4. A-Correlation between physical activity (PA) and academic performance (AP) of female students in private, public, rural and urban Schools. B-corresponding table illustration spearman correlation coefficient $(r)$ and goodness of fit $\left(r^{2}\right)$

Note. $n$ - number of students.

\section{DISCUSSION}

Increased physical activity has the potential to improve academic performance, as it increases the cognitive skills and academic behavior of students. It can also increase the concentration and attention, thereby improving classroom behavior (Centers for Disease Control and Prevention, 2010). Therefore, it can be postulated that PA has a positive influence on AP. This was re-enforced in the findings of our study, where we observed an association between PA and AP in school children aged 12-16 years, in two districts of Nepal. These results are supported by similar studies from various different cultures and countries which have also shown a positive association between AP and PA (Sardinha,
Marques, Martins, Palmeira, \& Minderico, 2014; So, 2012; Van Dusen, Kelder, Kohl, Ranjit, \& Perry, 2011). However, the academic performance and physical activity levels in our study population were associated in only some of the subgroups. There was a significant positive correlation between AP and PA among male students, students at public schools and students from both rural and urban areas.

However, analyzing the results through the gender lens, we found that PA of male students was positively correlated with AP irrespective of the location and type of schools. Whereas the PA of female students did not shown any correlation with AP. The non correlation between PA and AP 
in the female students could partly be explained by the finding that the female students were engaged in lesser PA than their male counterpart. In fact, another study done in Nepalese students have also shown that female students were less engaged in PA compared to male students (Paudel et al., 2014). Therefore, less PA could be one of the reasons that PA was unrelated to AP in the female students. Also, we found that the AP of male students was significantly better than their female counterparts. These findings showed that male students who were involved in more PA had better AP than their female counterparts who were involved in less PA. Thus, our results showed a plausible evidence of the involvement of PA in the improvement of AP. However, no definitive causation was established by our study and different socioeconomic variables must be considered before implying any causality.

Overall, our results showed that male students who were involved in higher physical activity also had a better academic performance. However, our study didn't take into account the effects of socioeconomic variables and psychometric variables that might have influenced our results. Having said that, our findings support, encourage and highlight the important role that PA may play in the AP of students in Nepal.

\section{CONCLUSIONS}

Physical activity forms a significant part of modern day life, regardless of age and gender. It is even more important for the well-being of the young population, not only for improving health, but also for ensuring an effective education. Our study found that females were physically less active than the males in the study population. Male students who were physically active than female students showed positive correlation between physical activity and academic performance, whereas no such correlation was seen in the female students. However, various socioeconomic variables need to be analyzed further before implying the causality.

Future Perspectives. This is the first study analyzing an association between physical activity levels and academic performance in Nepalese students. In Nepal, where studying is heavily prioritized over extracurricular activities, specifically sports, we hope that the results of this study will help open up a debate regarding the role and necessity of physical activity in school and students' overall academic performance.

Acknowledgments. We would like to extend our gratitude to the school administration of Shree Chandeshwori Secondary School and Kibou Boarding School in Dhading as well as Shree Rani Devi Secondary School and Takshyashila Boarding School in Kathmandu for allowing us to conduct our research. We are also indebted to the students who volunteered in our studies by providing their invaluable time.

Conflicts of interest - The authors declare no conflict of interest.

\section{REFERENCES}

Booth, J. N., Leary, S. D., Joinson, C., Ness, A. R., Tomporowski, P. D., Boyle, J. M., \& Reilly, J. J. (2013). Associations between objectively measured physical activity and academic attainment in adolescents from a UK cohort. British Journal of Sports Medicine, 48(3), 265-270. doi: 10.1136/bjsports-2013-092334

Carlson, S. A., Fulton, J. E., Lee, S. M., Maynard, L. M., Brown, D. R., Kohl, H. W., \& Dietz, W. H. (2008). Physical education and academic achievement in elementary school: Data from the early childhood longitudinal study. American Journal of Public Health, 98(4), 721-727. doi: 10.2105/ajph.2007.117176

Centers for Disease Control and Prevention, Department of Health and Human Services. (2010). The association between school based physical activity, including physical education, and academic performance. Retrieved from https:/www.cdc.gov/healthyyouth/ health_and_academics/pdf/pa-pe_paper.pdf

Correa-Burrows, P., Burrows, R., Ibaceta, C., Orellana, Y., \& Ivanovic, D. (2014). Physically active Chilean school kids perform better in language and mathematics. Health Promotion International. doi: 10.1093/heapro/dau010

Devkota, S. P., \& Bagale, S. (2015). Social inequality in Nepal and right of education. Journal of Poverty, Investment and Development, 8, 1-4.

Esteban-Cornejo, I., Tejero-González, C. M., MartinezGomez, D., Cabanas-Sánchez, V., FernándezSantos, J. R., Conde-Caveda, J., ... Veiga, O. L. (2014). Objectively measured physical activity has a negative but weak association with academic performance in children and adolescents. Acta Paediatrica, 103(11), e501-e506. doi: 10.1111/apa.12757

Fox, C. K., Barr-Anderson, D., Neumark-Sztainer, D., \& Wall, M. (2010). Physical activity and sports team participation: associations with academic outcomes in middle school and high school students. Journal of School Health, 80(1), 31-37. doi: 10.1111/j.17461561.2009.00454.x

Janak, J. C., Gabriel, K. P., Oluyomi, A. O., Perez, A., Kohl, H. W., 3rd, \& Kelder, S. H. (2014). The association 
between physical fitness and academic achievement in Texas state house legislative districts: an ecologic study. Journal of School Health, 84(8), 533-542. doi: 10.1111/ josh. 12176

Kowalski, K. C., Crooker, P. R. E., \& Donen, R. M. (2004). The physical activity questionnaire for older children (PAQ-C) and adolescents (PAQ-A) manual. College of Kinesiology, University of Saskatchewan, 87(1), 1-38.

LeBlanc, M. M., Martin, C. K., Han, H., Newton, R., Sothern, M., Webber, L. S., ... Williamson, D. A. (2012). Adiposity and physical activity are not related to academic achievement in school-aged children. Journal of Developmental and Behavioral Pediatrics, 33(6), 486-494. doi:10.1097/DBP.0b013e31825b849e

Paudel, S., Subedi, N., Bhandari, R., Bastola, R., Niroula, R., \& Poudyal, A. K. (2014). Estimation of leisure time physical activity and sedentary behaviour among school adolescents in Nepal. BMC Public Health, 14(1), 637. doi: 10.1186/1471-2458-14-637

Pokharel, A. K., \& Poudel, J. (2013). Cultural factors causing differences in quality education. Researcher: A Research Journal of Culture and Society, 1(2), 1-10. doi: http://dx.doi.org/10.3126/researcher.v1i2.9881

Sardinha, L. B., Marques, A., Martins, S., Palmeira, A., $\&$ Minderico, C. (2014). Fitness, fatness, and academic performance in seventh grade elementary school students. BMC Pediatrics, 14(176), 9. doi: 10.1186/1471-243114-176

Sharma, B. (1989-1992). Regional inequality in the size distribution of income in Nepal. The Himalayan Review, $X X-X X I I I, 23$.

Shin, Y. S., \& So, W. Y. (2012). Association between physical inactivity and academic record in korean adolescents. Iranian Journal of Public Health, 41(10), 36-42.
Singh, A., Uijtdewilligen, L., Twisk, J. W., van Mechelen, W., \& Chinapaw, M. J. (2012). Physical activity and performance at school: A systematic review of the literature including a methodological quality assessment. Archives of Pediatrics and Adolescent Medicine, 166(1), 49-55. doi: 10.1001/archpediatrics.2011.716

So, W.-Y. (2012). Association between physical activity and academic performance in Korean adolescent students. BMC Public Health, 12(1), 258. doi: 10.1186/1471-2458-12-258

Stea, T. H., \& Torstveit, M. K. (2014). Association of lifestyle habits and academic achievement in Norwegian adolescents: a cross-sectional study. BMC Public Health, 14(1), 829. doi: 10.1186/1471-2458-14-829

Taras, H. (2005). Physical activity and student performance at school. Journal of School Health, 75(6), 214-218. doi: 10.1111/j.1746-1561.2005.00026.x

United Nations Development Programme and National Planning Commission, Government of Nepal. (2014). Nepal Human Development Report 2014. Retrieved from www.hdr.undp.org/sites/default/files/nepal_ nhdr_2014-final.pdf

Van Dusen, D. P., Kelder, S. H., Kohl, H. W., 3rd, Ranjit, N., \& Perry, C. L. (2011). Associations of physical fitness and academic performance among schoolchildren. Journal of School Health, 81(12), 733740. doi: 10.1111/j.1746-1561.2011.00652.x

World Health Organization. (2017a). Global Strategy on Diet, Physical Activity and Health. Retrieved from http://www.who.int/dietphysicalactivity/factsheet_ recommendations/en/

World Health Organization. (2017b). Physical activity fact sheet. Retrieved from http://www.who.int/ mediacentre/factsheets/fs385/en/ 